\title{
Research Paper: The Relationship Between Different Forms of Maltreatment and Cognitive Emotion Regulation Strategies
}

\author{
Kamran Gorgi ${ }^{1}$ (D), Behrooz Dolatshai ${ }^{1 *}$ (D), Shima Shakiba1 ${ }^{10}$, Saeed Kamizi² ${ }^{(1)}$ \\ 1. Department of Clinical Psychology, University of Social Welfare and Rehabilitation Sciences, Tehran, Iran. \\ 2. Department of Clinical Psychology, Faculty of Psychology and Educational Sciences, Allameh Tabataba'i University, Tehran, Iran.
}

\begin{tabular}{|c|c|}
\hline $\begin{array}{l}\text { Use your device to scan } \\
\text { and read the article online }\end{array}$ & Chtation: Gorgi, K. Dolatshai, B. Shakiba, Sh. \& Kamizi, S. (2019). The Relationship Between Different Forms of Maltreat- \\
\hline aipla & $\begin{array}{l}\text { ment and Cognitive Emotion Regulation Strategies. Journal of Practice in Clinical Psychology, 7(4), 255-262. http://dx.doi. } \\
\text { org/10.32598/jpcp.7.4.255 }\end{array}$ \\
\hline 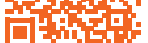 & dol'http://dx.doi.org/10.32598/jpcp.7.4.255 \\
\hline
\end{tabular}

\section{(i) (8)}

Article info:

Received: 21 Mar 2019

Accepted: 18 Aug 2019

Available Online: 01 Oct 2019

Keywords:

Physical abuse, Sexual abuse, Emotional abuse, Emotional regulation

\section{ABSTRACT}

Objective: The present study aimed to examine the relationship between different forms of maltreatment and cognitive emotion regulation strategies.

Methods: This research is a cross-sectional study. The sample included 321 individuals from the general population aged 18-50 years. They were selected from different districts of Tehran, using the convenience sampling method. The data were collected by the traumatic experiences checklist and the cognitive emotion regulation questionnaire and analyzed using the Pearson correlation coefficient and multivariate regression analysis and the enter method.

Results: Emotional maltreatment explained part of the variance of self-blame, rumination, catastrophizing, and blaming others $(\mathrm{P}<0.01)$. Physical maltreatment explained part of the variance of catastrophizing and blaming others $(\mathrm{P}<0.01)$, and sexual maltreatment had a significant positive correlation only with catastrophizing $(\mathrm{P}<0.05)$.

Conclusion: The results indicated a positive association between a history of maltreatment and the use of maladaptive emotion regulation strategies. Besides, it was found that various forms of maltreatment have different relationships with maladaptive cognitive emotion regulation strategies.

\section{* Corresponding Author:}

Behrooz Dolatshai, PhD

Address: Department of Clinical Psychology, University of Social Welfare and Rehabilitation Sciences, Tehran, Iran.

Tel: \pm 98 (917) 8202372

E-mail:dolatshahee@yahoo.com 


\section{Highlights}

- The Persian version of the MODDI-F has acceptable validity and reliability.

- A Cronbach's alpha of higher than 0.70 were found for all of the subscales of MODDI-F, expect for the subscale of death denial.

- A significant relationship was found between the scores of the MODDI-F components and the scores of convergent and divergent factors in the EAS, OHQ, GHQ, and DAS questionnaires.

\section{Plain Language Summary}

Many previous instruments about death were not based on true theory and well-explained conceptual assumptions. Besides, most of the existing instruments are only focused on anxiety related to death and dying. Few instruments are available to assess death acceptance, and none is appropriate for assessing the constructs of acceptance and fear of death in a multidimensional way. The Multidimensional Orientation Toward Dying and Death Inventory (MODDI-F) is the only instrument assessing death acceptance and death denial, along with death anxiety. The MODDI-F is based on the main assumption that people' reactions toward dying can be divided into three dimensions of fear, acceptance, and denial. These dimensions are the most important ways of thinking and feeling about death. MODDI-F consists of 8 subscales of fear of one's own dying, fear of one's own death, fear of another person's dying, fear of another person's death, fear of corpses, acceptance of one's own dying and death, acceptance of another person's death, and denial of one's own death. The present study aimed to examine the psychometric properties of the Persian version of the MODDI-F. Results showed the acceptable validity and reliability of its Persian version. A Cronbach's alpha of higher than 0.70 were found for all of the subscales of MODDI-F, expect for the subscale of death denial. A significant relationship was found between the scores of the MODDI-F components and the scores of convergent and divergent factors in the EAS, OHQ, GHQ, and DAS questionnaires.

\section{Introduction}

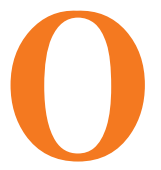

ne of the worst experiences of children is being subjected to maltreatment (Alink, Cicchetti, Kim, \& Rogosch, 2009). The National Society for the Prevention of Cruelty to Children defines maltreatment as all forms of physical and or emotional ill-treatment, sexual abuse, neglect or negligent treatment, or commercial or other exploitation, resulting in actual or potential harm to the child's health, survival, development or dignity in the context of a relationship of responsibility, trust, or power (Sawyerr, \& Bagley, 2017).

Maltreated children are at risk for various emotional and behavioral problems (Alink, Cicchetti, Kim, \& Rogosch, 2009) that may lead to such outcomes as interpersonal problems, eating disorders, suicide, and so on in adolescence and adulthood (Berzenski \& Yates, 2010). Generally, children who are maltreated use different strategies to manage their emotions; for example, they may use suppression or avoidance, try to reduce their emotional arousal using verbal or physical quarrels, experience dissociation, during which they automatically engage in imagination or depersonalizing, or have other reactions (Kirouac, 2009). Therefore, human beings can reduce, increase, or maintain their emotions consciously or unconsciously and adaptively or maladaptively (Gross, 2014).

The maturity of the brain parts related to emotion regulation is influenced by early experiences and caregiver's responsiveness (Dvir, Ford, Hill, \& Frazier, 2011). However, maltreating parents do not properly teach their children how to reduce their negative emotions; as a result, maltreated children tend to have deficiencies in emotion regulation that may lead to various disorders and problems. Many studies have shown the relationship between maltreatment and emotional dysregulation. For example, several studies report that those who had been subjected to emotional and behavioral maltreatment had lower scores on adaptive emotion regulation measures than those without such an experience (Alink, Cicchetti, Kim, \& Rogosch, 2009; Hartzell, 2009; Yarlasky, 2015). Based on another study, girls with a history of sexual abuse were less able to recognize and regulate their emotions (Dvir, Ford, Hill, \& Frazier, 2011). Among all forms of maltreatment, emotional maltreatment is the best predictor of 
emotion regulation problems (Hartzell, 2009; Dvir, Ford, Hill, \& Frazier, 2011).

The relationship between maltreatment and emotion regulation can also be explained from the neurobiological approach. For example, maltreatment leads to hemispheric asymmetry so that in these children, the right hemisphere has more activity than the left one (Alink, Cicchetti, Kim, \& Rogosch, 2009). In many studies, frontal lobe asymmetry with right-hemisphere dominance has been linked with depression and anxiety (Hostinar et al. 2017). Adverse early life experiences can change the activity threshold of the limbic system, which in turn, can affect the person's ability to perceive and evaluate threats (Dvir, Ford, Hill, \& Frazier, 2011). The mutual relationship between the immune system and the brain causes negative psychological experiences and longterm effects on mental health (Hostinar et al. 2017).

On the other hand, different forms of maltreatment lead to various symptoms. For example, in a previous study, the relationship between emotional neglect, sexual maltreatment, and behavioral maltreatment with 3 dimensions of depression and anxiety symptoms (general distress, anhedonic depression, and anxious arousal) was examined. The results indicated that emotional neglect was accompanied by 3 dimensions, behavioral maltreatment with two dimensions (general distress and anxious arousal), and physical maltreatment only with anxious arousal (Infurna et al., 2016). Another study suggested that different kinds of traumatic experiences had different impacts on emotion regulation. In other words, those with a history of traumatic experiences related to interpersonal relationships had more difficulty with emotion regulation than those with a history of traumatic experiences related to natural disasters (Kalil, 2012).

Although different factors at conscious to unconscious level are involved in emotion regulation (Gross, 2014), most studies, including the present one, have focused only on the conscious aspect of emotion regulation. Garnefski, Kraaij, and Spinhoven studied cognitive emotion regulation and developed the Cognitive Emotion Regulation Questionnaire (CERQ) that is used in the present study. They identified 9 cognitive emotion regulation strategies, among which 4 strategies are maladaptive strategies (self-blame, rumination, catastrophizing, and blaming others) and 5 are adaptive ones (putting into perspective, positive refocusing, positive reappraisal, acceptance, and refocus on planning). Individuals subjected to maltreatment may use one or several of these strategies. For example, in sexually-abused children, selfblame (e.g. "why I was not more careful?" or "why did I act like that?") is a common consequence related to shame and self-worthlessness (Ullman, Peter-Hagene, \& Relyea, 2015).

Different experiences of maltreatment adversely affect emotion regulation that is considered a fundamental factor for a psychological disorder. In this regard, it is essential to identify emotional regulation strategies that individuals use to cope with outcomes of trauma and to investigate this relationship and promote therapeutic approaches for victims of different injuries. Therefore, different experiences have different outcomes. Considering different adaptive and maladaptive cognitive emotion regulation strategies, the present study aimed at addressing the question of whether those who have experienced various forms of maltreatment use different cognitive emotion regulation strategies.

\section{Methods}

\section{Research design and participants}

The sample included 321 individuals from the general population, who were selected from north, south, east, and west of Tehran, using the convenience sampling method. The inclusion criteria included no history of head injury and using any current drug.The study questionnaires were distributed in parks and public places of Tehran City, Iran, among different groups. The study data were collected with the consent of the participants and by providing the required information for them.

Emotional, physical, and sexual traumas were measured by the Traumatic Experiences Checklist (TEC), which is a 2- item self-report questionnaire with good reliability and validity (Nijenhuis et al., 2002). The scores for the presence of both emotional trauma (emotional neglect and emotional abuse in various settings) and sexual trauma (sexual harassment and sexual abuse in multiple settings) were based on 6 items. The scores for the presence of physical abuse in various settings were based on 3 items. This phrase preceded all items: did this happen to you? An example of a sexual abuse item is sexual abuse (unwanted sexual acts involving physical contact) by your parents, brothers, or sisters. For all 3 types of interpersonal trauma, a dichotomous score (yes/ no) was used. In the present study, the reliability of TEC was found as 0.72 .

CERQ was developed both on a theoretical and empirical basis to identify the cognitive coping strategies individuals use after experiencing threatening or stressful events (Garnefski et al., 2001). It has 36 items and assesses 9 different cognitive emotion regulation strate- 
gies. The strategies are divided into 2 categories; more adaptive strategies, including acceptance, positive reappraisal, positive refocusing, refocusing on planning, and putting into perspective, as well as less adaptive strategies, including self-blame, blaming others, catastrophizing, and rumination. The scores of each strategy can range from 4-20. For the total scale score, alpha value reliabilities of $0.91,0.87$, and 0.93 were found (Garnefski et al., 2001). In Iran, the Cronbach alpha for the total scale score was 0.82 . In the present study, the reliability values of the adaptive and maladaptive strategies were 0.84 and 0.74 , respectively.

The Pearson correlation coefficient and multiple regression analysis using the Enter method were used to analyze the data. All analyses were performed in SPSS V. 20.

\section{Results}

Table 1 includes the results of the Pearson correlation coefficient for the relationship between different dimensions of maltreatment and maladaptive cognitive emotion regulation strategies. According to Table 1, there are significant relationships between various forms of maltreatment and maladaptive cognitive emotion regulation strategies.
In other words, there are significant positive correlations between emotional and behavioral maltreatment and all maladaptive cognitive emotion regulation strategies, and sexual maltreatment have a significant positive relationship only with catastrophizing. Therefore, higher scores on different forms of maltreatment are related to a higher possibility of using maladaptive cognitive emotion regulation strategies. On the other hand, no significant correlation was found between maltreatments and adaptive cognitive emotion regulation strategies.

Table 2 presents the results of multivariate regression analysis for different forms of maltreatment predicting the use of maladaptive cognitive emotion regulation strategies.

Three forms of maltreatment predict $20.7 \%$ of the variance of maladaptive cognitive emotion regulation strategies (Table 2) $(\mathrm{P}<0.1 ; \mathrm{F}=28.870)$.

In addition, emotional maltreatment explained part of the variance of self-blame $(\mathrm{P}<0.01$, Beta $=0.307)$, rumination $(\mathrm{P}<0.01$, Beta $=0.269)$, catastrophizing $(\mathrm{P}<0.01$, Beta $=0.272)$, and blaming others $(\mathrm{P}<0.01$, Beta $=0.143)$. Also, physical maltreatment explained part of the variance of catastrophizing $(\mathrm{P}<0.01$, Beta $=0.146)$ and blaming others $(\mathrm{P}<0.01$, Beta $=0.126)$. Different forms of mal-

Table 1. Correlation coefficients between maladaptive cognitive emotion regulation strategies and 3 forms of maltreatment

\begin{tabular}{|c|c|c|c|c|}
\hline Variables & $\mathbf{R}$ & $\mathbf{P}$ & $\mathbf{P}$ & $\mathbf{N}$ \\
\hline \multirow{3}{*}{ Self-Blaming } & PA & $0.189 *$ & 0.001 & 321 \\
\hline & SA & 0.089 & 0.113 & 321 \\
\hline & EA & $0.332^{*}$ & 0.000 & 321 \\
\hline \multirow{3}{*}{ Rumination } & PA & $0.137^{* *}$ & 0.014 & 321 \\
\hline & SA & 0.050 & 0.372 & 321 \\
\hline & EA & $0.276^{*}$ & 0.000 & 321 \\
\hline \multirow{3}{*}{ Catastrophizing } & PA & $0.270^{*}$ & 0.000 & 321 \\
\hline & SA & $0.113^{* *}$ & 0.043 & 321 \\
\hline & EA & $0.342^{*}$ & 0.000 & 321 \\
\hline \multirow{3}{*}{ Other-blaming } & PA & $0.198^{*}$ & 0.000 & 321 \\
\hline & SA & 0.109 & 0.051 & 321 \\
\hline & EA & $0.211^{*}$ & 0.000 & 321 \\
\hline \multirow{3}{*}{ Total maladaptive EM } & PA & $0.306^{*}$ & 0.000 & 321 \\
\hline & SA & $0.139 * *$ & 0.013 & 321 \\
\hline & EA & $0.445^{*}$ & 0.000 & 321 \\
\hline \multirow{3}{*}{ Total adaptive EM } & PA & 0.044 & .432 & 321 \\
\hline & SA & -0.106 & 0.057 & 321 \\
\hline & EA & 0.104 & 0.062 & 321 \\
\hline
\end{tabular}

* Correlation is significant at the 0.01 level (2-tailed);

** Correlation is significant at the 0.05 level (2-tailed). 
Table 2. Regression coefficients for different forms of maltreatment predicting the use of maladaptive cognitive emotion regulation strategies

\begin{tabular}{|c|c|c|c|c|c|c|c|}
\hline Dependent V & Predictor V & Beta & $\mathbf{T}$ & $\mathbf{P}$ & Adjusted R Square & $\mathbf{F}$ & $\mathbf{P}$ \\
\hline & PA & 0.053 & 0.897 & 0.371 & & & \\
\hline \multirow[t]{3}{*}{ Self-blaming } & SA & 0.011 & 0.196 & 0.845 & 0.105 & 13.454 & 0.000 \\
\hline & EA & 0.307 & 5.144 & 0.000 & & & \\
\hline & PA & 0.022 & 0.362 & 0.718 & & & \\
\hline \multirow[t]{3}{*}{ Rumination } & SA & -0.014 & -0.257 & 0.798 & 0.068 & 8.751 & 0.000 \\
\hline & EA & 0.269 & 4.423 & 0.000 & & & \\
\hline & PA & 0.146 & 2.513 & 0.012 & & & \\
\hline \multirow[t]{3}{*}{ Catastrophizing } & SA & 0.026 & 0.492 & 0.623 & 0.128 & 16.591 & 0.000 \\
\hline & EA & 0.272 & 4.624 & 0.000 & & & \\
\hline & PA & 0.126 & 2.082 & 0.038 & & & \\
\hline \multirow[t]{3}{*}{ Other-blaming } & SA & 0.055 & 0.987 & 0.324 & 0.052 & 6.903 & 0.000 \\
\hline & EA & 0.143 & 2.333 & 0.020 & & & \\
\hline & $\mathrm{PA}$ & 0.135 & 2.431 & 0.016 & & & \\
\hline \multirow[t]{2}{*}{ Total maladaptive EM } & SA & 0.031 & 0.596 & 0.551 & 0.207 & 28.870 & 0.000 \\
\hline & EA & 0.380 & 6.760 & 0.000 & & & \\
\hline
\end{tabular}

treatment can explain a significant part of the variance of cognitive emotion regulation.

\section{Discussion}

Child maltreatment can have negative and vast consequences. Emotional regulation ability is one of the most critical areas damaged by maltreatment. The question arises whether those who have experienced different forms of maltreatment tend to use different emotion regulation strategies.

Concerning the lack of association of adaptive strategies of emotional regulation with different kinds of maltreatment, the results of this study are in line with previous studies (Huh, Kim, Lee, \& Chae, 2017), illustrating that maladaptive strategies are related to psychological trauma; however, adaptive strategies have less correlation with different types of maltreatment. In some studies, the relationship between emotional neglect and adaptive strategies has been observed. These studies refer to social learning theory and explain being not a suitable pattern to learn adaptive strategies. It can explain the relationship between neglect and the use of adaptive strategies. In this study, there was no such relationship.

Overall, there was an association between different forms of maltreatment experienced and the use of maladaptive cognitive emotion regulation strategies; more specifically, different forms of maltreatment have different relationships with different kinds of maladaptive cognitive emotion regulation strategies. This finding is in line with the previous findings showing different consequences for different forms of maltreatment. For example, Briere and Runtz (1990) showed that a history of emotional maltreatment is related to low self-esteem, and a history of physical maltreatment is related to aggression toward others. Lobbestael, Arntz, and Bernstein (2010) also found different relationships between different forms of maltreatment and various personality disorders. For example, emotional maltreatment was related to paranoid, schizotypal, borderline, and cluster $\mathrm{C}$ personality disorders. Physical maltreatment was related to antisocial personality disorder, and sexual maltreatment was related to paranoid, schizoid, borderline, and avoidant personality disorders. Kinard also reported that 
different forms of maltreatment were related to various aspects of behavioral performance.

In general, we can point out some reasons (such as emotional, cognitive, and motivational ones) why people choose different emotion regulation strategies. Regarding the emotional aspect, the intensity of emotion makes the person choose different strategies; for example, when the intensity of negative emotion is very high, distraction is probably used more than reappraisal. Regarding the cognitive aspect and depending on the cognitive complexity of the experience, different strategies are selected. Concerning the motivational aspect and depending on how likely it is that the person experiences an emotion again, different strategies are used; for example, when it is expected that the person frequently experiences the emotion, reappraisal could have a more prolonged effect than distraction that could be the preferred choice if the possibility of experiencing the emotion was not more than once (Sheppes \& Levin, 2013). Given that various personal characteristics and experiences make people prefer specific strategies (Backus, 2013), and considering that there are different forms of maltreatment, we can expect that people choose different strategies, and that tendency toward some strategies could be stronger than others. For example, those with a history of emotional maltreatment are more likely to engage in self-blame as a maladaptive strategy, perhaps because, in emotional maltreatment, the destructive message has a more transparent and more direct influence on the self-worthiness and self-efficacy of the child. This is probably the reason that emotional maltreatment leads to depression more than the other forms of maltreatment (Hartzell, 2009). In addition to the form of maltreatment, when the maltreatment occurs, its frequency, intensity, etc. also influence the outcomes (Kinard, 2004).

In the present study, despite the differences observed in the relationships among different forms of maltreatment and various emotion regulation strategies, the correlations were relatively low. The present research focused on the conscious aspect of emotion regulation. In other words, we relied on self-report data; however, emotional phenomena could also be present in the absence of conscious awareness (Berlin, 2001). Those who have an insecure attachment have a low ability to realize and connect to their negative emotions (Becker-Stoll, Delius, Stepha, 2001). Therefore, an implicit or unconscious part exists in each person inaccessible through introspection . Besides, conscious assessments are usually affected by the social desirability bias, in which respondents try to describe themselves better than what they are (Hofmann, Gawronski, Gschwendner, \& Schmitt, 2005). This is probably the reason why strong correlations were not found in the present study. Therefore, we suggest that future studies examine unconscious emotion regulation, mainly because very few studies have compared the impact of different forms of maltreatment.

The participants with more maltreatment experiences have more problems in emotion regulation. This study focuses on several practical implications, including the importance of the relationship between maltreatment experiences with emotional regulation in mental health studies. This can increase the awareness of experts in this field about the consequences of maltreatment. In addition, researchers and therapists in this field will consider the maltreatment a risk factor for the defect in regulating emotions and mental disorders.

\section{Ethical Considerations}

\section{Compliance with ethical guidelines}

All ethical principles were considered in this article. The participants were informed about the purpose of the research and its implementation stages; they were also assured about the confidentiality of their information; Moreover, They were allowed to leave the study whenever they wish, and if desired, the results of the research would be available to them.

\section{Funding}

This research did not receive any specific grant from funding agencies in the public, commercial, or not-forprofit sectors.

\section{Authors' contributions}

All authors contributed equally in preparing all parts of the research.

\section{Conflict of interest}

The authors declared no conflict of interest.

\section{References}

Alink, L. R., Cicchetti, D., Kim, J., \& Rogosch, F. A. (2009). Mediating and moderating processes in the relation between maltreatment and psychopathology: Mother-child relationship quality and emotion regulation. Journal of Abnormal Child Psychology, 37(6), 831-43. [DOI:10.1007/s10802-009-9314-4] [PMID] [PMCID] 
Backus, F. (2013). The relationship between cognitive emotion regulation and clinical symptoms: A gendered analysis [PhD. dissertation]. Chestnut Hill, MA: Boston College.

Becker-Stoll, F., Delius, A., \& Scheitenberger, S. (2001). Adolescents' nonverbal emotional expressions during negotiation of a disagreement with their mothers: An attachment approach. International Journal of Behavioral Development, 25(4), 344-53. [DOI:10.1080/01650250143000102]

Berlin, H. A. (2011). The neural basis of the dynamic unconscious. Neuropsychoanalysis, 13(1), 63-71. [DOI:10.1080/15294 145.2011.10773663]

Berzenski, S. R., \& Yates, T. M. (2010). A developmental process analysis of the contribution of childhood emotional abuse to relationship violence. Journal of Aggression, Maltreatment $\mathcal{E}$ Trauma, 19(2), 180-203. [DOI:10.1080/10926770903539474]

Briere, J., \& Runtz, M. (1990). Differential adult symptomatology associated with three types of child abuse histories. Child Abuse \& Neglect, 14(3), 357-64. [DOI:10.1016/01452134(90)90007-G]

Dvir, Y., Ford, J. D., Hill, M., \& Frazier, J. A. (2014). Childhood maltreatment, emotional dysregulation, and psychiatric comorbidities. Harvard Review of Psychiatry, 22(3), 149-61. [DOI:10.1097/HRP.0000000000000014] [PMID] [PMCID]

Garnefski, N., Kraaij, V., \& Spinhoven, P. (2001). Negative life events, cognitive emotion regulation and emotional problems. Personality and Individual Differences, 30(8), 1311-27. [DOI:10.1016/S0191-8869(00)00113-6]

Gross, J. J. (2014). Emotion regulation: Conceptual and empirical foundations. In J. J. Gross (Ed.), Handbook of emotion regulation (pp. 3-22). $2^{\text {nd }}$ ed. New York: Guilford Publications.

Hartzell, E. E. (2009). Child maltreatment, emotion regulation, and posttraumatic stress [MSc. thesis]. Athens, GA: University of Georgia.

Hofmann, W., Gawronski, B., Gschwendner, T., Le, H., \& Schmitt, M. (2005). A meta-analysis on the correlation between the Implicit Association Test and explicit self-report measures. Personality and Social Psychology Bulletin, 31(10) 1369-85. [DOI:10.1177/0146167205275613] [PMID]

Hostinar, C. E., Davidson, R. J., Graham, E. K., Mroczek, D. K., Lachman, M. E., \& Seeman, T. E., et al. (2017). Frontal brain asymmetry, childhood maltreatment, and low-grade inflammation at midlife. Psychoneuroendocrinology, 75, 152-63. [DOI:10.1016/j.psyneuen.2016.10.026] [PMID] [PMCID]

Huh, H. J., Kim, K. H., Lee, H. K., \& Chae, J. H. (2017). The relationship between childhood trauma and the severity of adulthood depression and anxiety symptoms in a clinical sample: The mediating role of cognitive emotion regulation strategies. Journal of Affective Disorders, 213, 44-50. [DOI:10.1016/j. jad.2017.02.009] [PMID]

Infurna, M. R., Reichl, C., Parzer, P., Schimmenti, A., Bifulco, A., $\&$ Kaess, M. (2016). Associations between depression and specific childhood experiences of abuse and neglect: A meta-analysis. Journal of Affective Disorders, 190, 47-55. [DOI:10.1016/j. jad.2015.09.006] [PMID]

Kalill, K. S. (2012). The role of difficulties in emotion regulation in the relationship between experiences of trauma and later dissociation [PhD. dissertation]. Boston, MA: University of Massachusetts Boston.
Kinard, E. M. (2004). Methodological issues in assessing the effects of maltreatment characteristics on behavioral adjustment in maltreated children. Journal of Family Violence, 19(5), 303-18. [DOI:10.1023/B:JOFV.0000042080.98027.ae]

Kirouac, S., \& McBride, D. L. (2009). The impact of childhood trauma on brain development: A literature review and supporting handouts. Retrieved from https://eric. ed.gov/?id=ED512316

Lobbestael, J., Arntz, A., \& Bernstein, D. P. (2010). Disentangling the relationship between different types of childhood maltreatment and personality disorders. Journal of Personality Disorders, 24(3), 285-95. [DOI:10.1521/pedi.2010.24.3.285] [PMID]

Milojevich, H. M. (2016). The role of maltreatment in the development of emotion regulation [PhD. dissertation]. Irvine, CA: University of California Irvine.

Nijenhuis, E. R. S., Van der Hart, O., \& Kruger, K. (2002). The psychometric characteristics of the Traumatic Experiences Checklist (TEC): First findings among psychiatric outpatients. Clinical Psychology \& Psychotherapy, 9(3), 200-10. [DOI:10.1002/ cpp.332]

Sawyerr, A., \& Bagley, C. (2017). Child sexual abuse and adolescent and adult adjustment: A review of British and world evidence, with implications for social work, and mental health and school counselling. Advances in Applied Sociology, 7(1), 1-15. [DOI:10.4236/aasoci.2017.71001]

Sheppes, G., \& Levin, Z. (2013). Emotion regulation choice: Selecting between cognitive regulation strategies to control emotion. Frontiers in Human Neuroscience, 7, 179. [DOI:10.3389/ fnhum.2013.00179] [PMID] [PMCID]

Ullman, S. E., Peter-Hagene, L. C., \& Relyea, M. (2014). Coping, emotion regulation, and self-blame as mediators of sexual abuse and psychological symptoms in adult sexual assault. Journal of Child Sexual Abuse, 23(1), 74-93. [DOI:10.1080/10538 712.2014.864747] [PMID] [PMCID]

Yarlasky, K. (2015). Childhood sexual abuse, adolescent substance use and the moderating role of emotion regulation [MA. thesis]. London, ON: Western University. 
This Page Intentionally Left Blank 